\title{
Lumbosacral Transitional Vertebra-Related Low Back Pain: Resolving the Controversy
}

\author{
Balachandar Gopalan, Janardhan Srinivas Yerramshetty \\ Department of Orthopaedics, Chettinad Hospital and Research Institute, Kancheepuram, India
}

\begin{abstract}
Study Design: Case control study.
Purpose: The association of lumbosacral transitional vertebra (LSTV) with low back pain (LBP) is controversial, as is the role of occupational physical activity and radiological spinal abnormalities suggestive of other spinal disorders (OSDs) such as spinal degeneration and instability. This study aimed to determine if any association of LSTV with LBP exists. If so, the association of the level of physical activity and presence of OSD with LSTV-related LBP was determined.

Overview of Literature: The cause of LBP has been linked to proximal level disc degeneration, arthritic pseudoarticulation between LSTV and the sacral ala, facet joint degeneration, and nerve root compression due to a broadened transverse process. LSTV associated with LBP is present among individuals who are involved in high-level physical activity, including military recruits and athletes.

Methods: This was an unmatched study comprising 372 cases and 224 controls consecutively recruited with clinical and radiographic documentation. The relationship between LSTV and LBP was analyzed, and the effects of LSTV and OSD on this relationship were also assessed and statistically controlled.

Results: The presence of LSTV ( $p=0.039$ ) was significantly associated with LBP, and the presence of OSD was associated with LTSVrelated LBP, after statistically controlling for the level of physical activity $(p=0.024)$. The level of physical activity was not associated with LBP. Demographic analysis revealed female predominance with an advanced age ( $>45$ years) among those with LSTV-related LBP who have OSD.

Conclusions: The presence of LSTV was associated with an increased prevalence of LBP. This association was probably due to the confounding effect of OSD. The level of occupational physical activity was not associated with LSTV-related LBP. We speculate that advanced age and female sex caused the spurious association of LSTV with LBP in our study, rendering LSTV-related LBP controversial in published literature.
\end{abstract}

Keywords: Lumbosacral transitional vertebra; Low back pain; Physical activity; Lumbar spondylosis; Spinal instability

\section{Introduction}

Lumbosacral transitional vertebra (LSTV) is a congenital anomaly of the spine that arises because of mutations in the Hox genes, giving rise to sacralization (fifth lumbar vertebra shows assimilation to the sacrum) and lumbarization (first sacral vertebra shows lumbar configuration).

Spinal disorders (concurrent or secondary) that are commonly associated with LSTV [1] are spinal degeneration and spinal instability. A nine times higher risk for

Received Jul 5, 2017; Revised Oct 18, 2017; Accepted Oct 30, 2017

Corresponding author: Balachandar Gopalan

Department of Orthopedics, Al Qassimi Hospital, Sharjah, United Arab Emirates

Tel: +971-6-518-8888, Fax: +971-6-538-7200, E-mail: drbala8782@gmail.com 
disc degeneration suprajacent to LSTV [2] and altered function of lumbar nerve roots [3] were associated with LSTV, whereas spinal canal stenosis [4] and spondylolisthesis [5] were not associated with LSTV. Evidence vary with regard to the association of pars lysis with LSTV; Otani et al. [6] have shown a positive correlation, whereas Elster [2] have shown no correlation between the two entities.

Perhaps the most controversial aspect is the association of LSTV with low back pain (LBP). Studies showing positive correlation implicate disc herniation or degeneration proximal to LSTV [7], disc herniation compressing the nerve roots [8], arthritic pseudoarticulation between LSTV and the sacral ala [8], facet joint contralateral to the fused/articulating LSTV [9], or extraforaminal stenosis secondary to a broadened transverse process [10]. In contrast, some studies have also demonstrated no association of LSTV with LBP [11,12]. Luoma et al. [12] studied 138 middle-aged men ( $40-45$ years) and 25 young asymptomatic men (18-20 years) and reported no association of transitional vertebra with any type of LBP among the middle-aged men. They also reported that the disc below the transitional vertebra was protected from degeneration among the middle-aged men, whereas the disc above the transitional vertebra showed signs of degeneration among the young asymptomatic men. Tini et al. [11] studied 4,000 lumbar radiographs in patients with LBP and 1,873 patients without LBP. They reported the incidences of LSTV as $6.7 \%$ and $5 \%$, respectively, and concluded that LSTV was not related to LBP.

The influence of physical activity on LBP has not been extensively studied, albeit the presence of conflicting evidence. Riihimaki et al. [13] conducted a cross-sectional study among men aged $25-49$ years with sciatic pain involved in three different types of work, namely machine operation, construction carpentry, and office work. The former two groups represented dynamic work, while the latter represented sedentary work. They concluded that machine operators and carpenters were more susceptible to sciatic pain than sedentary workers. However, Videman et al. [14], in their cross-sectional study comprising 600 men aged 35-70 years, showed that occupational loading and heavier work were associated with less disc desiccation, which was beneficial for the lumbar spine.

LSTV is reported to be associated with LBP among individuals involved in high-level physical activity such as military recruits and athletes. Taskaynatan et al. [15] reported a series of 881 young male military recruits with LBP of $>4$ weeks duration, of whom 48 had LSTV. They concluded that transitional vertebra was associated with LBP and radicular pain. Mann et al. [16] reported on a 20-year-old baseball player with LBP for 1 year and leftsided radiculopathy who underwent the excision of the enlarged transverse process of L5 on the left side, which was impinging on the sacrum. After temporary pain relief for 12 weeks, the patient complained of non-radicular LBP with radiographic evidence of reformation of the enlarged L5 transverse process. Back et al. [17] reported on two golfers with chronic LBP in whom the source of pain was identified to be the sacrotransverse joint of LSTV through diagnostic imaging and fluoroscopic injections; one of them was treated with radiofrequency denervation along the pseudoarticulation and the other received ipsilateral sacrotransverse intraarticular and L5 transforaminal epidural injection with good pain relief.

We frequently observed that patients with LBP and radiological evidence suggestive of other spinal disorders (OSDs), such as spondylosis or instability, also had LSTV. We also noted that several of them were laborers. We aimed to determine any association of LSTV with LBP and whether this was related to OSD and/or the level of physical activity.

\section{Materials and Methods}

Informed written consent and the institutional review board approval of Chettinad Hospital and Research Institute, Kelambakkam, Tamilnadu for the study were obtained (ethical committee approval no., IHEC/02/2013/ Desp No 208-24.4.2013). A total of 372 consecutive patients attending the orthopedic outpatient clinic of Chettinad Hospital and Research Institute (Kelambakkam, Tamilnadu, India), a tertiary care university hospital with a history of LBP of $>4$ weeks were recruited between February 2013 and August 2014. Patients with a clinical evidence of paraspinal muscle spasm, positive straight leg raise test, and neurological deficits (motor/sensory/reflex) were included. Patients with pain suggestive of traumatic, infective, inflammatory, abdominopelvic, or renal origin and those having back pain with a history of previous surgeries in the spine and multiple surgeries involving spinal anesthesia were excluded. The quality of LBP was categorized as mild (pain that can be easily ignored), moderate (pain that cannot be ignored, interferes with function, and 
needs attention or treatment from time to time), severe (pain that is present most of the time, demanding constant attention or treatment), and excruciating (totally incapacitating pain) [18]. Only patients with moderate and severe pain were included because they were more representative of a clinical picture of patients seeking care at an outpatient setting. Patients with mild LBP were symptomatically managed and observed with follow-up visits. If they returned and the LBP was moderate or severe, then they were subjected to further imaging and then included in the study. Patients with excruciating pain and significant nerve root tension signs with gross sensorimotor deficit usually presented to the emergency department and were excluded from the study.

\section{Occupational data}

A detailed occupational history was recorded to categorize the patients into the high- and low-activity groups. The high-activity group (laborers) included farmers and laborers who worked at construction sites and performed activities such as plastering, concrete cutting, piping, masonry, and carpentry. These individuals were involved in carrying heavy weight and/or repetitive actions involving the flexion and extension of the lumbar spine, working for 10-12 hours a day for at least 1 year. The low-activity group (non-laborers) included those working in less physically demanding environments, such as software professionals, office workers, and housewives.

\section{Imaging}

Radiographs of the lumbar spine (anteroposterior [AP] and lateral views) and cervicothoracic spine (which enabled accurate numbering from $\mathrm{C} 2$ vertebra, AP view) were obtained. The morphology of transverse processes on the AP view of the lumbosacral spine enabled the classification of LSTV (Fig. 1A-D) [4]. Ferguson (30 cephalad) view was obtained to measure the width of the dysplastic transverse process (the vertical length at the junction of the middle and lateral thirds as described by Ohmori et al. [19]) only when the AP radiograph revealed enlargement but not beyond the threshold of 19 $\mathrm{mm}$. On standing lateral radiographs of the lumbosacral spine, the height of the disc was calculated as the average of the sum of the distance between the anterior, middle, and posterior intervertebral disc spaces of the last mobile (proximal to LSTV) and last formed (distal to LSTV) levels, except in type $3 b$ (complete fusion of sacrotransverse joint, bilateral) where there is a rudimentary disc space distally [20]. Different types of LSTV are explained in Fig. 1. Type 0 represents a fully lumbarised vertebra (L6) with normal transverse processes. Disc height narrowing was considered significant when the disc height was $>-2$ standard deviation from the mean. OSD, defined as radiological findings suggestive of spondylosis (facet arthrosis, segmental instability, traction spurs, osteophytes, disc height narrowing, degenerative spondylolisthesis, and degenerative scoliosis) and instability (pars lysis and lytic
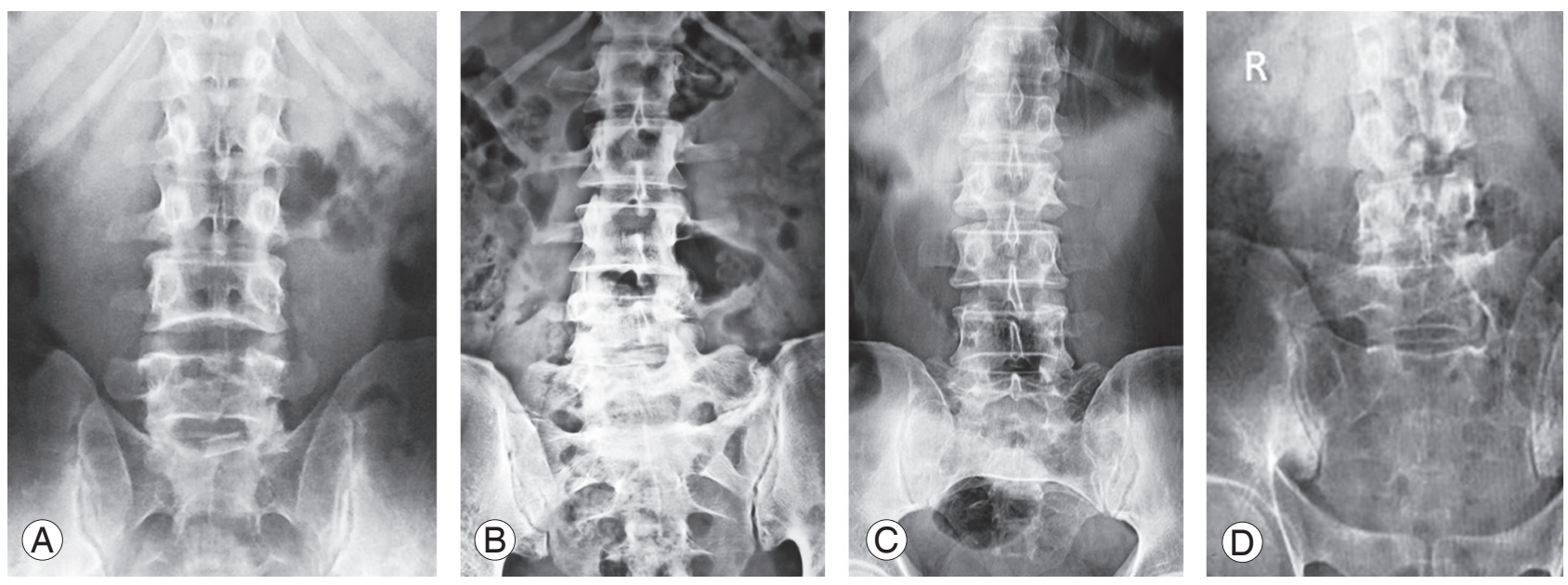

Fig. 1. (A) Type 1 b: dysplastic transverse process ( $\geq 19-\mathrm{mm}$ width). (B) Type 2b: incomplete sacralization/lumbarization (pseudoarthrosis between the transverse process with adjacent sacral ala). (C) Type 3b: complete sacralization/lumbarization (completely fused transverse process and adjacent sacral ala). (D) Type 4: mixed (type 2 on one side and type 3 on the other). Type a: unilateral. Type b: bilateral. 
spondylolisthesis) was documented. Segmental instability was defined as the antero- or retrolisthesis of the proximal vertebra by $>8 \%$ relative to the distal vertebra on neutral standing radiograph [21]. Degenerative spondylolisthesis was defined as the forward slip of vertebra relative to the distal vertebra, which was associated with facet joint(s) degeneration or reduced disc space. Lytic spondylolisthesis was defined as the forward slip of vertebra relative to the distal vertebra with pars defect. Degenerative scoliosis was defined as the lumbar curvature on the frontal plane $\geq 10^{\circ}$ to either side associated with degenerative changes [22]. Other spinal radiographic findings that are not well known to be associated with LBP, such as spina bifida occulta, limbus vertebra, and Schmorl nodes, were recorded but not analyzed.

\section{Controls}

A control group of 224 patients attending the orthopedic outpatient department without LBP but with non-spinal musculoskeletal complaints were included. This group included patients with and without LSTV. These patients were grouped on the basis of recorded occupational data. Patients with a history of polytrauma/multiple fractures with suspected injuries to the spine with or without operative interventions on follow-up, those with history of os- teoporosis with or without treatment, those with a history of medical illnesses such as pancreatitis and renal stones (mimicking LBP), and those with axial pain in the neck or midback and asymptomatic patients previously treated for LBP regardless of etiology were excluded. Spine radiographs of patients were obtained after acquiring informed consent.

To understand the confounding effects of the relationship between LSTV and LBP, patients were further classified on the basis of activity (high and low) and OSD with regard to LBP. To avoid any bias from the high-activity group, only the low-activity group was selected to assess the relationship between OSD and LSTV. Similarly, to avoid any bias in relation to OSD, only the OSD-absent group was selected to assess the relationship between activity and LSTV. Finally, to eliminate the interference with regard to activity and OSD, the relationship between LSTV and LBP was analyzed within the subgroup that comprised low-activity and OSD-absent patients.

\section{Statistical methods}

Continuous variables were tested for the normality of distribution and analyzed using Student $t$-test. Categorical variables were analyzed using the chi-square test or Fisher's exact test. Statistical significance was set at $p \leq 0.05$.

Table 1. Demographics of cases and controls among 596 subjects

\begin{tabular}{|c|c|c|c|c|}
\hline Characteristic & Cases $(n=372)$ & Controls ( $n=224$ ) & Total & $p$-value \\
\hline Age (yr) & $37.2(16-74)$ & 34 (17-66) & & $0.001^{\mathrm{a})}$ \\
\hline Sex & & & & $<0.001^{\text {b) }}$ \\
\hline Male & $211(56.6)$ & $161(43.3)$ & 373 & \\
\hline Female & $162(72.3)$ & $62(27.7)$ & 223 & \\
\hline Sacralization & $88(70.4)$ & $37(29.6)$ & 125 & \\
\hline Lumbarization & $13(65)$ & $7(35)$ & 20 & \\
\hline LSTV type & & & & $0.009^{c)}$ \\
\hline Type 0 & $3(42.9,3)$ & $4(57.1,9.1)$ & 7 & \\
\hline Type 1 & $17(58.6,16.8)$ & $12(41.4,27.3)$ & 29 & \\
\hline Type 2 & $36(73.5,35.6)$ & $13(26.5,29.5)$ & 49 & \\
\hline Type 3 & $45(78.9,44.6)$ & $12(21.1,27.3)$ & 57 & \\
\hline Type 4 & $0(0,0)$ & $3(100.0,6.8)$ & 3 & \\
\hline Total & 101 & 44 & 145 & \\
\hline
\end{tabular}

Values are presented as mean (min-max), number (\%), or mean\% (row-wise, column-wise), unless otherwise stated. In the LSTV subtype classification, row- and column-wise percentages are depicted in parentheses.

LSTV, lumbosacral transitional vertebra.

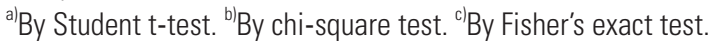




\section{Results}

The demographics of the 596 patients are shown in Table 1. The mean age of cases was higher than that of controls (mean difference, 3.26 years; 95\% confidence interval [CI], $1.4-5.1 ; p=0.001)$. There were more males among the cases $(35.4 \%$ versus $27 \%)$ and controls (27\% versus $10 \%)$ in the overall sample. Women were twice likely to have LBP than men (odds ratio [OR], 1.99; 95\% CI, 1.4-2.8; $p<0.001)$. The prevalence of LSTV was $24.3 \%$ (145/596). Sacralization $(125 / 596,20.9 \%)$ was approximately six times more frequent than lumbarization (20/596,3.3\%), with predominance of types $3 \mathrm{~b}(48 / 145)$ and $2 \mathrm{a}(37 / 145)$ in the overall sample as well as in both groups. There were seven lumbarized vertebrae with normal transverse processes (LSTV type 0 ). The incidence of hypoplasia or aplasia of the 12th rib was significantly high in those with LSTV $(67 / 145,46.2 \%)$ than in those without LSTV (39/451, 8.6\%). On plain radiographs, numerous spinal radiological findings were observed, and their distribution is depicted in Table 2. Among the 372 patients with LBP, mild motor deficits were present in five (two from the high-activity group and three from the low-activity group), sensory hypoesthesia in 17 (seven from the highactivity group and 10 from the low-activity group), and positive straight leg raise test in 14 (three from the highactivity group and 11 from the low-activity group).

Analysis on the complete sample of 596 patients re- vealed a significant association of LBP with LSTV (OR, 1.52; 95\% CI, 1.02-2.27; $p=0.039$ ), the presence of OSD (OR, 5.43; 95\% CI, 2.65-11.1; $p<0.001$ ), and the lowactivity group (OR, 1.76; 95\% CI, 1.2-2.5; $p=0.002$ ) (Table 3). To avoid any bias with regard to the presence of OSD and high-level physical activity, both of which have been reported to be associated with LSTV-related LBP, the re-

Table 2. Distribution of radiological findings among cases and controls Radiological findings Cases $(n=372) \quad$ Controls $(n=224)$

\begin{tabular}{lll} 
Spondylolisthesis $^{\text {a) }}$ & 29 & 0 \\
Degenerative scoliosis $^{\text {b) }}$ & 10 & 0 \\
\hline Disc height narrowing $^{\text {b) }}$ & 14 & 1 \\
Facet joint arthrosis $^{\text {b) }}$ & 2 & 0 \\
Traction spurs $^{\text {b) }}$ & 4 & 3 \\
Segmental instability $^{\text {b) }}$ & 7 & 0 \\
\hline Osteophytosis $^{\text {b) }}$ & 3 & 0 \\
Pars defect $^{\text {a) }}$ & 5 & 2 \\
Schmorl node $^{c)}$ & 4 & 6 \\
Spina bifida $^{c)}$ & 4 & 0 \\
Limbus vertebra $^{c)}$ & 1 & 3
\end{tabular}

Instability signs and degenerative signs represent other spinal disorders.

a)Instability signs: they include pars defect (fusion defect in pars interarticularis) and lytic spondylolisthesis (defined as the forward slip of vertebra relative to the distal vertebra with pars defect). ${ }^{\text {b) }}$ Degenera-

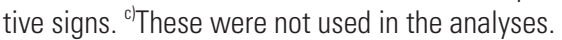

Table 3. Distribution of LSTV, OSDs, and level of physical activity in the entire sample

\begin{tabular}{|c|c|c|c|c|}
\hline Within entire sample & Cases (\%) & Controls (\%) & Total & $p$-value ${ }^{\text {a) }}$ \\
\hline LSTV & & & & 0.039 \\
\hline Non-LSTV & $271(60.1)$ & $180(39.9)$ & 451 & \\
\hline LSTV & $101(69.7)$ & 44 (30.3) & 145 & \\
\hline Total & 372 & 224 & 596 & \\
\hline OSD & & & & $<0.001$ \\
\hline Present & 69 (88.5) & $9(11.5)$ & 78 & \\
\hline Absent & $303(58.5)$ & $215(41.5)$ & 518 & \\
\hline Total & 372 & 224 & 596 & \\
\hline Activity & & & & 0.002 \\
\hline High & $101(53.2)$ & $89(46.8)$ & 190 & \\
\hline Low & $271(66.7)$ & $135(33.3)$ & 406 & \\
\hline Total & 372 & 224 & 596 & \\
\hline
\end{tabular}

LSTV, lumbosacral transitional vertebra; OSD, other spinal disorder.

${ }^{a}$ By chi-square test. 
Table 4. Relationship between OSD and LSTV in the low-activity group and activity and LSTV in the OSD-absent group

\begin{tabular}{|c|c|c|c|c|}
\hline Variable & Non-LSTV (\%) & LSTV (\%) & Total & $p$-value ${ }^{\text {a) }}$ \\
\hline Within low-activity group & & & & 0.024 \\
\hline OSD absent & $269(77.1)$ & $80(22.9)$ & 349 & \\
\hline OSD present & $36(63.2)$ & $21(36.8)$ & 57 & \\
\hline Total & 305 & 101 & 406 & \\
\hline Within OSD-absent group & & & & 0.969 \\
\hline High activity & $130(76.9)$ & $39(23.1)$ & 169 & \\
\hline Low activity & $269(77.1)$ & $80(22.9)$ & 349 & \\
\hline Total & 399 & 119 & 518 & \\
\hline
\end{tabular}

OSD, other spinal disorder; LSTV, lumbosacral transitional vertebra.

a) By chi-square test.

Table 5. Relationship between LSTV and low back pain after the removal of confounding effects of high activity and OSD

\begin{tabular}{lcccc} 
Low-activity and OSD-absent group & Cases $(\%)$ & Controls (\%) & Total & $p$-value ${ }^{\text {a) }}$ \\
Non-LSTV & $164(61)$ & $105(39)$ & 269 & 0.289 \\
LSTV & $54(67.5)$ & $26(32.5)$ & 349 \\
\hline Total & 218 & 131 & 0 \\
\hline
\end{tabular}

LSTV, lumbosacral transitional vertebra; OSD, other spinal disorder.

a) By chi-square test.

Table 6. Age and sex distribution among the 16 subgroups (A-P)

Activity group LSTV OSD No. of patients Age (yr) as mean (range) Sex (male:female) as absolute (\%)

\begin{tabular}{|c|c|c|c|c|c|c|}
\hline \multicolumn{7}{|c|}{ Case } \\
\hline$A$ & High & No & No & 63 & $31.7(18-70)$ & $60: 3$ (95.2:4.8) \\
\hline B & High & No & Yes & 12 & 46 (32-62) & $7: 5(58.3: 41.7)$ \\
\hline C & High & Yes & No & 22 & $34.7(16-58)$ & $20: 2(91: 9)$ \\
\hline$D$ & High & Yes & Yes & 4 & $37.7(30-41)$ & 4:0 (100:0) \\
\hline$E$ & Low & No & No & 164 & $36(17-68)$ & $81: 83(49.3: 50.7)$ \\
\hline $\mathrm{F}$ & Low & No & Yes & 32 & $43.7(20-64)$ & 9:23 (28:72) \\
\hline G & Low & Yes & No & 54 & $37.2(19-65)$ & $25: 29(46.3: 53.7)$ \\
\hline $\mathrm{H}$ & Low & Yes & Yes & 21 & $50.7(29-74)$ & $5: 16(24: 76)$ \\
\hline \multicolumn{7}{|c|}{ Control } \\
\hline I & High & No & No & 67 & $31.6(20-55)$ & $59: 8$ (88:12) \\
\hline$J$ & High & No & Yes & 4 & $49.5(38-60)$ & $3: 1(75: 25)$ \\
\hline K & High & Yes & No & 17 & $34.6(20-59)$ & $16: 1(94: 6)$ \\
\hline $\mathrm{L}$ & High & Yes & Yes & 1 & 65 & $1: 0(100: 0)$ \\
\hline$M$ & Low & No & No & 105 & $33.3(17-66)$ & $64: 41$ (61:39) \\
\hline $\mathrm{N}$ & Low & No & Yes & 4 & $51.7(40-65)$ & 4:0 (100:0) \\
\hline 0 & Low & Yes & No & 26 & 35.7 (19-64) & $15: 11(57.7: 42.3)$ \\
\hline$P$ & Low & Yes & Yes & 0 & 0 & 0 \\
\hline
\end{tabular}

LSTV, lumbosacral transitional vertebra; OSD, other spinal disorder; M, male; F, female. 
Table 7. Mean age and sex distribution among various pairs of variables

\begin{tabular}{|c|c|c|c|c|c|c|c|}
\hline \multirow[b]{2}{*}{ Variable } & \multicolumn{3}{|c|}{ Age } & \multicolumn{4}{|c|}{ Sex } \\
\hline & $\begin{array}{c}\text { Mean age } \\
(y r)\end{array}$ & $\begin{array}{c}\text { Mean difference } \\
(95 \% \mathrm{Cl})\end{array}$ & $p$-value ${ }^{\text {a) }}$ & Male & Female & $\begin{array}{l}\text { Odds ratio } \\
(95 \% \mathrm{CI})\end{array}$ & $p$-value ${ }^{\text {b) }}$ \\
\hline Low activity (n=406) & 37.02 & $3.14(1.2-5.1)$ & 0.002 & $203(50)$ & $203(50)$ & $8.44(5.1-14.1)$ & $<0.001$ \\
\hline High activity (n=196) & 33.87 & & & $170(86.7)$ & $20(13.3)$ & & \\
\hline LSTV (n=145) & 38.45 & $3.23(1.1-5.3)$ & 0.003 & $86(59.3)$ & $59(40.7)$ & NS & NS \\
\hline Non-LSTV (n=451) & 35.24 & & & $287(63.6)$ & $164(36.4)$ & & \\
\hline OSD present (n=78) & 46.64 & $12.24(9.7-14.7)$ & $<0.001$ & $33(42.3)$ & $45(57.7)$ & $2.6(1.6-4.2)$ & $<0.001$ \\
\hline OSD absent ( $n=518)$ & 34.42 & & & $340(65.6)$ & $178(34.4)$ & & \\
\hline
\end{tabular}

Values are presented as number or number (\%), unless otherwise stated.

Cl, confidence interval; LSTV, lumbosacral transitional vertebra; NS, not significant; OSD, other spinal disorder.

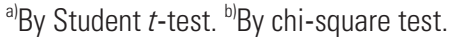

lationships between LSTV and OSD (in the low-activity group) and LSTV and level of activity (in the OSD-absent group) were analyzed (Table 4). Finally, analysis between LSTV and LBP was performed in the low-activity and OSD-absent group (Table 5). The presence of OSD in the low-activity group was statistically significant in relation to LBP. The level of physical activity had no association with LSTV-related LBP.

Although the relationship between LSTV and LBP could be interpreted as spurious because of the presence of OSD, it was counterintuitive to find this in the lowactivity group rather than in the high-activity group. Thus, cases and controls were distributed in terms of age and sex ratio among three variables (LSTV, OSD, and level of physical activity) (Table 6). Female predominance was observed among the low-activity group with OSD, irrespective of the presence or absence of LSTV, whereas male predominance was observed in all other groups. Analysis was performed in the overall sample with respect to age and sex (Table 7). The presence of OSD was significantly associated with female predominance and advanced age (>45 years), whereas the presence of LSTV was not associated with sex or age.

\section{Discussion}

A major reason for the polarity of conclusions regarding LSTV and LBP in previous studies was their recruitment of a highly specific target population, e.g., young males $[7,8]$, young females [4], elderly individuals [23], male laborers $[13,14,24]$, those with LBP only without a control group [21,25], those without LBP [4], and a lack of analysis in the control group [12]. The problem with such studies is that the lack of an appropriate comparative or control group makes the impact of results less valuable because they do not represent the entire spectrum of the general population. For instance, Taskaynatan et al. [15], in their prospective study of 881 military recruits, reported that the presence of transitional vertebra (48/881, 5.4\%) worsens the clinical picture and increases the nerve root symptoms regardless of the presence or absence of a causal relationship. However, they did not have a control group of asymptomatic patients or a comparative group comprising low-activity individuals to investigate the distribution of LSTV. A comparative study was conducted by Dr. Macnab, who reviewed the lumbar spine radiographs of three hundred 40-year-old laborers (150 without LBP and 150 with LBP treatment). No difference was found in the distribution of anatomical variants and degenerative changes [24], which is consistent with our finding. However, we identified an altered distribution of OSD among non-laborers, i.e., the low-activity group.

Few studies have analyzed the effect of age and sex on LSTV. Bertolotti's syndrome (transitional vertebra with LBP) has been considered by some to predominantly occur among the younger population [6,7]. However, our patients with LSTV were slightly older than those without LSTV, which is not clinically significant. Nardo et al. [23] reported that LSTV was more prevalent in men, whereas Sekharappa et al. [26] found an increased incidence among women. Ucar et al. [25] reported no differences in the distribution of LSTV with respect to age and sex. We did not find sexual dimorphism in the prevalence of LSTV. 
The relationship between spinal degenerative/instability disorders and increased age is intuitive because of age-related degeneration. This is also evident in our data wherein those with OSD were usually almost a decade older than those without OSD. The relationship between sex and OSD is less well understood. Females experience worse LBP, with associated depression [27] and metabolic syndrome [28]. Unemployed and married females performing household activities have reported worse LBP [28]. Strowbridge [29] reported that females engaged in physical military training experienced more LBP than men, which was not observed in women who played sports. Taylor et al. [30] identified 5,690 patients with degenerative lumbosacral pathologies and reported a greater prevalence of instability, spondylosis, and spinal stenosis among females and of herniated nucleus pulposus among males. Liu et al. [26], in their cross-sectional study on 7,075 patients, reported that scoliosis was more commonly found in females and the elderly. This is consistent with our results where spondylosis, instability, and scoliosis as well as LBP were found to be more prevalent among females (despite an overall male predominance in the entire sample).

Our study presented several advantages. First, data was prospectively collected from consecutive patients, which enabled accuracy and completeness and eliminated recall bias. Second, the standardized use of the cervicothoracic spine among all patients revealed that nearly half of those with LSTV had 12th rib hypoplasia, which could easily be mistaken for L1 vertebra on plain lumbar spine radiographs. The clinical implication of LSTV with regard to the correct numbering cannot be overemphasized, particularly in light of surgical interventions for spinal disorders.

This study also has some limitations. MRI would have detected more spinal abnormalities such as annular tears, disc herniations, end-plate changes, and spinal or foraminal stenosis, which would have provided a better estimate of the distribution of OSD. However, financial constraints and its absolute indication only in the presence of supportive clinical features made this modality expendable to our study. Radiological features of disc degeneration, such as reduced disc space, traction spurs, osteophytes, facet arthrosis, and segmental instability, served as surrogate markers in the absence of MRI. Flexion-extension radiographs are more appropriate in detecting segmental instability, but they were not obtained in all patients be- cause of the risk of an additional unnecessary radiation exposure (particularly in controls) and its relatively minor role in treatment. Further, although all radiographs were reviewed on multiple occasions at different time points (by BG, first author), intraobserver variability was not measured. Finally, further exploration of data would have been possible if the history of smoking, level of education, body mass index, and psychological status were available for all patients.

\section{Conclusions}

LSTV is associated with LBP. The presence of OSD is associated with LSTV-related LBP, whereas the level of physical activity is not associated with LBP. Furthermore, female sex is independently associated with LBP and OSD, whereas advanced age ( $>45$ years) is associated with OSD. Our study gives an indication to the dichotomy in literature regarding the association of LSTV with LBP because proponents may have overlooked the role of age, sex, or OSD, whereas opponents may have negated their confounding role altogether by targeting specific populations.

\section{Conflict of Interest}

No potential conflict of interest relevant to this article was reported.

\section{References}

1. Jancuska JM, Spivak JM, Bendo JA. A review of symptomatic lumbosacral transitional vertebrae: Bertolotti’s syndrome. Int J Spine Surg 2015;9:42.

2. Elster AD. Bertolotti's syndrome revisited: transitional vertebrae of the lumbar spine. Spine (Phila $\mathrm{Pa}$ 1976) 1989;14:1373-7.

3. Chang HS, Nakagawa H. Altered function of lumbar nerve roots in patients with transitional lumbosacral vertebrae. Spine (Phila Pa 1976) 2004;29:1632-5.

4. Oguz H, Akkus S, Tarhan S, Acikgozoglu S, Kerman M. Measurement of spinal canal diameters in young subjects with lumbosacral transitional vertebra. Eur Spine J 2002;11:115-8.

5. Cinotti G, Postacchini F, Fassari F, Urso S. Predisposing factors in degenerative spondylolisthesis: a radiographic and CT study. Int Orthop 1997;21:337-42. 
6. Otani K, Konno S, Kikuchi S. Lumbosacral transitional vertebrae and nerve-root symptoms. J Bone Joint Surg Br 2001;83:1137-40.

7. Quinlan JF, Duke D, Eustace S. Bertolotti's syndrome: a cause of back pain in young people. J Bone Joint Surg Br 2006;88:1183-6.

8. Jonsson B, Stromqvist B, Egund N. Anomalous lumbosacral articulations and low-back pain: evaluation and treatment. Spine (Phila Pa 1976) 1989;14:831-4.

9. Brault JS, Smith J, Currier BL. Partial lumbosacral transitional vertebra resection for contralateral facetogenic pain. Spine (Phila Pa 1976) 2001;26:226-9.

10. Shibayama M, Ito F, Miura Y, Nakamura S, Ikeda S, Fujiwara K. Unsuspected reason for sciatica in Bertolotti’s syndrome. J Bone Joint Surg Br 2011;93:705-7.

11. Tini PG, Wieser C, Zinn WM. The transitional vertebra of the lumbosacral spine: its radiological classification, incidence, prevalence, and clinical significance. Rheumatol Rehabil 1977;16:180-5.

12. Luoma K, Vehmas T, Raininko R, Luukkonen R, Riihimaki H. Lumbosacral transitional vertebra: relation to disc degeneration and low back pain. Spine (Phila Pa 1976) 2004;29:200-5.

13. Riihimaki H, Viikari-Juntura E, Moneta G, Kuha J, Videman T, Tola S. Incidence of sciatic pain among men in machine operating, dynamic physical work, and sedentary work: a three-year follow-up. Spine (Phila Pa 1976) 1994;19:138-42.

14. Videman T, Levalahti E, Battie MC. The effects of anthropometrics, lifting strength, and physical activities in disc degeneration. Spine (Phila Pa 1976) 2007;32:1406-13.

15. Taskaynatan MA, Izci Y, Ozgul A, Hazneci B, Dursun $\mathrm{H}$, Kalyon TA. Clinical significance of congenital lumbosacral malformations in young male population with prolonged low back pain. Spine (Phila Pa 1976) 2005;30:E210-3.

16. Mann DC, Keene JS, Drummond DS. Unusual causes of back pain in athletes. J Spinal Disord 1991;4:33743.

17. Back JD, Wyss JF, Lutz GE. Bertolotti syndrome as a potential cause of low back pain in golfers. PM R 2011;3:771-5.

18. Solomon L, Wakeley C. Diagnosis in orthopaedics. In: Solomon L, Warwick D, Nayagam S, editors. Apley's system of orthopaedics and fractures. 9th ed. London: Hodder Arnold; 2010. p.3-4.
19. Ohmori K, Ishida Y, Takatsu T, Inoue H, Suzuki K. Vertebral slip in lumbar spondylolysis and spondylolisthesis: long-term follow-up of 22 adult patients. J Bone Joint Surg Br 1995;77:771-3.

20. Inoue H, Ohmori K, Miyasaka K, Hosoe H. Radiographic evaluation of the lumbosacral disc height. Skeletal Radiol 1999;28:638-43.

21. Leone A, Guglielmi G, Cassar-Pullicino VN, Bonomo L. Lumbar intervertebral instability: a review. Radiology 2007;245:62-77.

22. Liu G, Tan JH, Ee G, Chan YH, Low SL, Wong HK. Morphology and prevalence study of lumbar scoliosis in 7,075 multiracial Asian adults. J Bone Joint Surg Am 2016;98:1307-12.

23. Nardo L, Alizai H, Virayavanich W, et al. Lumbosacral transitional vertebrae: association with low back pain. Radiology 2012;265:497-503.

24. De Luzzi M. Spondylogenic backache: soft tissue lesions and pain mechanisms. In: Wong DA, Transfeldt E, editors. Macnab's backache. 4th ed. Philadelphia (PA): Lippincott Williams \& Wilkins; 2007. p.60-90.

25. Ucar BY, Ucar DE, Bulut M, Azboy I, Demirtas A. Lumbosacral transitional vertebrae in low back pain population. J Spine 2013;2:1000125. https://doi. org/10.4172/2165-7939.1000125.

26. Sekharappa V, Amritanand R, Krishnan V, David KS. Lumbosacral transition vertebra: prevalence and its significance. Asian Spine J 2014;8:51-8.

27. Chenot JF, Becker A, Leonhardt C, et al. Sex differences in presentation, course, and management of low back pain in primary care. Clin J Pain 2008;24:578-84.

28. Ono R, Yamazaki S, Takegami M, et al. Gender difference in association between low back pain and metabolic syndrome: locomotive syndrome and health outcome in Aizu cohort study (LOHAS). Spine (Phila Pa 1976) 2012;37:1130-7.

29. Strowbridge NF. Gender differences in the cause of low back pain in British soldiers. J R Army Med Corps 2005;151:69-72.

30. Taylor BA, Casas-Ganem J, Vaccaro AR, Hilibrand AS, Hanscom BS, Albert TJ. Differences in the work-up and treatment of conditions associated with low back pain by patient gender and ethnic background. Spine (Phila Pa 1976) 2005;30:359-64. 